\title{
Fuzzy Brain Emotional Cerebellar Model Articulation Control System Design for Multi- Input Multi-Output Nonlinear
}

\author{
Chang-Chih Chung', Chih-Min Lin $^{2}$ \\ ${ }^{1}$ Department of Electrical Engineering, Yuan Ze University, Chung-Li, Tao-Yuan \\ 320, Taiwan, Republic of China \\ ${ }^{2}$ School of Information Science and Engineering, Xiamen University, China, and \\ Department of Electrical Engineering and Innovation Center for Big Data and \\ Digital Convergence, Yuan Ze University, Chung-Li, Tao-Yuan 320, Taiwan, \\ Republic of China, E-mail: cml@ saturn.yzu.edu.tw
}

\begin{abstract}
A brain emotional cerebellar model articulation controller (BECMAC) is developed, which is a mathematical model that approximates the judgmental and emotional activity of a brain. A fuzzy inference system is incorporated into the BECMAC, to give the novel fuzzy brain emotional cerebellar model articulation controller (FBECMAC) that is also proposed in this paper. The developed FBECMAC has the benefit of fuzzy inference and judgment and emotional activity, and it is used to control multi-input multi-output nonlinear systems. A 3-dimensional (3D) chaotic system and a mass spring damper mechanical system are simulated, to illustrate the effectiveness of the proposed control method. A comparison between the proposed FBECMAC and other controller shows that the proposed controller exercises better control than the other controllers.
\end{abstract}

Keywords: brain emotional cerebellar model articulation controller; fuzzy system; chaotic system; mass spring damper mechanical system

\section{Introduction}

In 1992, LeDoux found that, in the human brain, the association between a stimulus and its emotional consequence occurs in the amygdala [1]. The brain has an orbitofrontal cortex and an amygdala; the former is a sensory neural network and the latter is an emotional neural network [2]. Many works studied the form of the amygdala to determine the usefulness for a neural network control system. In recent years, brain emotional learning controllers (BELC) have been used for control systems by several studies [3-7]. A brain emotional learning controller has two systems: an emotional system and a neural network judgment system. 
A cerebellar model articulation controller (CMAC) is a network model that uses an associated memory network [8]. It has better computation and adaptation ability than a neural network. CMAC approximation can be tuned for greater accuracy, even for complex nonlinear system. Therefore, CMAC has been the subject of more studies, because it is more generally applicable to various nonlinear systems and can learn rapidly. Chiang and Lin introduced a Gaussianbased CMAC with faster convergence $[9,10]$. This gives more option for the researcher to experiment with a Gaussian-based CMAC to control various nonlinear systems. The enhanced performance of a CMAC over a neural network has been demonstrated in some studies $[11,12]$. By constructing a BELC using a CMAC, a new brain emotion network, called a brain emotion CMAC (BECMAC), is proposed. This improves the learning ability of a conventional BELC.

A fuzzy inference system mimics the human reasoning process and is widely used successfully in various fields. Initially, the control system algorithms required a detailed system model. However, in recent years, fuzzy control systems have used fuzzy inference rules to control systems, without the need for detailed mathematical models [13, 14].

This paper incorporates a fuzzy inference system with a BECMAC, to produce a novel fuzzy brain emotional cerebellar model articulation controller (FBECMAC). This controller has the benefits of a fuzzy system, because there are fuzzy inference rules, and of BECMAC, because the controller learns more completely. This FBECMAC is then used to control multi-input multi-output nonlinear systems, to illustrate its effectiveness.

\section{Problem Formulation}

A class of $n$-th order multi-input multi-output nonlinear systems is described by the following equation:

$$
\boldsymbol{x}^{(n)}(t)=\boldsymbol{f}(\underline{\boldsymbol{x}}(t))+\boldsymbol{G}(\underline{\boldsymbol{x}}(t)) \boldsymbol{u}(t)+\boldsymbol{d}(t)
$$

where

$$
\boldsymbol{u}(t)=\left[u_{1}(t), u_{2}(t), \cdots, u_{m}(t)\right]^{T} \in \mathfrak{R}^{m}
$$
$\boldsymbol{x}(t)=\left[x_{1}(t), x_{2}(t), \cdots, x_{m}(t)\right]^{T} \in \mathfrak{R}^{m}$. The former is a control input and the latter represents the state vectors of the system. $\boldsymbol{d}(t)=\left[d_{1}(t), d_{2}(t), \cdots, d_{m}(t)\right]^{T} \in \mathfrak{R}^{m}$ denotes the unknown bounded external disturbance and $m$ is the number of system inputs and outputs. $\underline{\boldsymbol{x}}(t)=\left[\boldsymbol{x}^{T}(t), \dot{\boldsymbol{x}}^{T}(t), \cdots, \boldsymbol{x}^{(n-1) T}(t)\right]^{T} \in \mathfrak{R}^{m n}$ and it is assumed that it is measureable. It is also true that $\boldsymbol{f}(\underline{\boldsymbol{x}}(t)) \in \mathfrak{R}^{m}$ and $\boldsymbol{G}(\underline{\boldsymbol{x}}(t)) \in \mathfrak{R}^{m \times m}$ and that these are smooth nonlinear uncertain functions, which are assumed to be bounded, but not exactly known. 
If modeling uncertainties and external disturbance are neglected, the nominal system for (1) is:

$\boldsymbol{x}^{(n)}(t)=\boldsymbol{f}_{0}(\underline{\boldsymbol{x}}(t))+\boldsymbol{G}_{0} \boldsymbol{u}(t)$

where $\boldsymbol{f}_{0}(\underline{\boldsymbol{x}}(t)) \in \mathfrak{R}^{m}$ and $\boldsymbol{G}_{0}=\operatorname{diag}\left(g_{01}, g_{02}, \cdots, g_{0 m}\right) \in \mathfrak{R}^{m \times m}$ are the nominal parts of $\boldsymbol{f}(\underline{\boldsymbol{x}}(t))$ and $\boldsymbol{G}(\underline{\boldsymbol{x}}(t))$, respectively. Without loss of generality, it is assumed that the constants $g_{0 \mathrm{i}} \geq 0$ for $i=1, \cdots, m$. It is also assumed that the nonlinear system (2) is controllable and that $\boldsymbol{G}_{0}^{-1}$ exists. If there are modeling uncertainties and external disturbances, the nonlinear system (1) can be reformulated as

$\boldsymbol{x}^{(n)}(t)=\boldsymbol{f}_{0}(\underline{\boldsymbol{x}}(t))+\boldsymbol{G}_{0} \boldsymbol{u}(t)+\boldsymbol{l}(\underline{\boldsymbol{x}}(t), t)$

where $\boldsymbol{l}(\underline{\boldsymbol{x}}(t), t)$ is referred to as the lumped uncertainty, which includes the system uncertainties and the external disturbances.

The control problem is the design of a proper control system, wherein the system output, $\boldsymbol{x}(t)$, can track a desired trajectory vector, $\boldsymbol{x}_{r}(t)=\left[x_{r 1}(t), x_{r 2}(t), \cdots, x_{r m}(t)\right]^{T} \in \mathfrak{R}^{m}$.

The tracking error is defined as

$\boldsymbol{e}(t) \stackrel{\Delta}{=} \boldsymbol{x}_{d}(t)-\boldsymbol{x}(t) \in \mathfrak{R}^{m}$

and the system tracking error vector is defined as

$\underline{\boldsymbol{e}}(t) \triangleq\left[\boldsymbol{e}^{T}(t), \dot{\boldsymbol{e}}^{T}(t), \cdots, \boldsymbol{e}^{(n-1) T}(t)\right]^{T} \in \mathfrak{R}^{m n}$

If the nominal functions, $f_{0}(\underline{x}(t)), \boldsymbol{G}_{0}$ and the lumped uncertainty, $\boldsymbol{l}(\underline{\boldsymbol{x}}(t), t)$, are exactly known, an ideal controller can be designed as:

$$
\boldsymbol{u}^{*}=\boldsymbol{G}_{0}^{-1}\left[\boldsymbol{x}_{d}^{(n)}-\boldsymbol{f}_{0}(\underline{\boldsymbol{x}})-\boldsymbol{l}(\underline{\boldsymbol{x}}, t)+\underline{\boldsymbol{K}}^{T} \underline{\boldsymbol{e}}\right]
$$

where $\underline{K}=\left[\boldsymbol{K}_{n}, \cdots, \boldsymbol{K}_{2}, \boldsymbol{K}_{1}\right]^{T} \in \mathfrak{R}^{m \times x m}$ is the feedback gain matrix, which contains real numbers, and $\boldsymbol{K}_{i}=\operatorname{diag}\left(k_{i 1}, k_{i 2}, \ldots k_{i n}\right) \in \mathfrak{R}^{m \times m}$ is a nonzero positive constant diagonal matrix.

Substituting the ideal controller (6) into (3) gives the error dynamic equation:

$\boldsymbol{e}^{(n)}+\underline{\boldsymbol{K}}^{T} \underline{\boldsymbol{e}}=\mathbf{0}$

In (7), if $\underline{K}$ is chosen to correspond to the coefficients of a Hurwitz polynomial, then $\lim _{t \rightarrow \infty}\|\underline{\boldsymbol{e}}\|=0$. However, the lumped uncertainty, $\boldsymbol{l}(\underline{\boldsymbol{x}}(t), t)$, is generally 
unknown for practical applications, so the ideal controller, $u^{*}$, in (6) is not possible. Therefore, a fuzzy BECMAC that mimics this ideal controller is proposed in the next section.

\section{A Fuzzy Brain Emotion Cerebellar Model Articulation Control System}

\subsection{The Fuzzy Brain Emotional Cerebellar Model Articulation Controller}

A fuzzy BECMAC (FBECMAC) control system can be classified as a supervised network. A FBECMAC is not very complex in operation and has fast convergence, so it is applicable to many nonlinear control systems. The proposed FBECMAC is shown in Fig. 1, and it has two systems; the first system is a propinquity amygdala system, similar to that in a mammalian brain; and the second system is a propinquity cerebellar system, which is also similar to that in a mammalian brain. In this novel inference system, two fuzzy rule bases are proposed for the BECMAC.

The fuzzy amygdala system is designed as:

If $I_{1}$ is $\lambda_{1}$ and $\ldots I_{i}$ is $\lambda_{i}, \ldots$,and $I_{m}$ is $\lambda_{m}$ then

$a_{q}=z_{i q}$ for $i=1,2, \ldots, m, q=1,2, \ldots, p$

where $z_{i q}$ is the amygdala's weight and $a_{q}$ is the amygdala's output.

The fuzzy cerebellar model articulation system is designed as:

If $I_{1}$ is $\phi_{1 j}$ and $\ldots I_{i}$ is $\phi_{i j}, \ldots$, and $I_{m}$ is $\phi_{m j}$ then

$o_{q}=w_{j q}$ for $i=1,2, \ldots, m, j=1,2, \ldots, n, q=1,2, \ldots, p$

where $w_{j q}$ is the prefrontal weight and $o_{q}$ is the prefrontal output. 


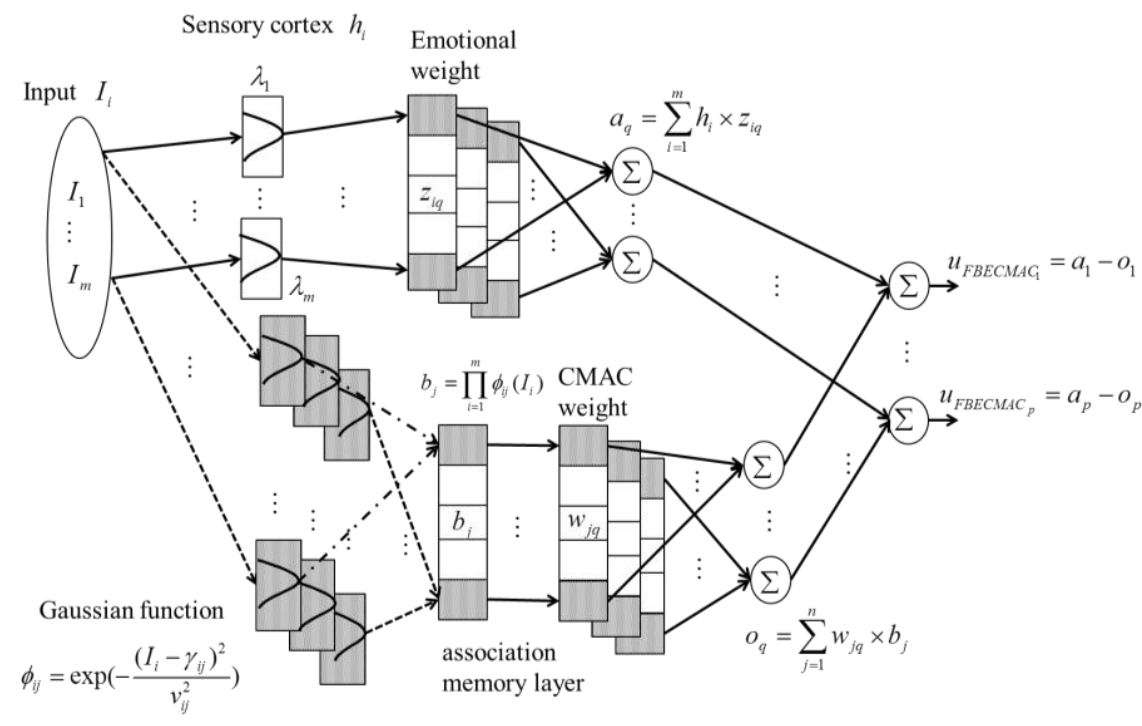

Figure 1

The emotional cerebellar model articulation controller

Fuzzy amygdala systems have two layers. The first layer is a Gaussian function and the second layer is a weight layer:

$h_{i}=I_{i} \times \lambda_{i}, i=1,2, \ldots, m$

where $h_{i}$ is the amygdala system's input to the sensory cortex output, $I_{i}$ is the controller's input and $\lambda_{i}$ is the Gaussian function, which is denoted as:

$\lambda_{i}=\exp \left(-\frac{\left(I_{i}-\delta_{i}\right)^{2}}{\sigma_{i}^{2}}\right)$

where $\delta_{i}$ is a mean and $\sigma_{i}$ is a variance.

$a_{q}=\sum_{i=1}^{m} h_{i} \times z_{i q}$

where $z_{i q}$ is amygdala system weight.

The fuzzy cerebellar model articulation system has three layers. The first layer is a Gaussian function:

$\phi_{i j}=\exp \left[-\frac{\left(I_{i}-\gamma_{i j}\right)^{2}}{v_{i j}^{2}}\right], i=1,2, \ldots, m, j=1,2, \ldots, n$

where $\gamma_{i j}$ is the mean and $v_{i j}$ is the variance. The second layer is an association memory layer: 
$b_{j}=\prod_{i=1}^{m} \phi_{i j}\left(I_{i}\right)$

The third layer is a weight layer:

$o_{q}=\sum_{j=1}^{n} w_{j q} \times b_{j}$

where $w_{j q}$ is a weight.

$u_{\text {FBECMAC }_{q}}=a_{q}-o_{q}, q=1,2 \ldots p$

The amygdala's system's updated weights, $\Delta z_{i q}$, are given by

$\Delta z_{i q}=\eta_{z}\left[h_{i} \times\left(\max \left[0, d_{q}-a_{q}\right]\right)\right]$

where $\eta_{z}$ is the learning rate. In (17), $d_{q}$ is a parameter adjustment, given by:

$d_{q}=\left(\sum_{i=1}^{m} \beta_{i q} \times I_{i}\right)+\left(c_{q} \times u_{F B E C M A C_{q}}\right)$

where $\beta_{i q}$ and $c_{q}$ are the gains.

The updating law for the amygdala's system's weight is given by

$z_{i q}(t+1)=z_{i q}(t)+\Delta z_{i q}$

The fuzzy CMAC hypercube weight, $w_{j q}$, and the mean, $m_{i j}$, and variance, $v_{i j}$, of the Gaussian function are updated using the following equation:

$w_{j q}(t+1)=w_{j q}(t)+\Delta w_{j q}$

$\gamma_{i j}(t+1)=\gamma_{i j}(t)+\Delta \gamma_{i j}$

$v_{i j}(t+1)=v_{i j}(t)+\Delta v_{i j}$

An integrated error function is defined as

$$
\boldsymbol{s}(\underline{\boldsymbol{e}}, t) \equiv \boldsymbol{e}^{(n-1)}+\boldsymbol{K}_{1} \boldsymbol{e}^{(n-2)}+\ldots+\boldsymbol{K}_{n} \int_{0}^{t} \boldsymbol{e}(\tau) d \tau
$$

where $\boldsymbol{s}(\underline{\boldsymbol{e}}, t)=\left[s_{1}(t), s_{2}(t), \cdots, s_{m}(t)\right]^{T} \in \mathfrak{R}^{m}$.

Substituting (3) into (23) yields

$\dot{\boldsymbol{s}}(\underline{\boldsymbol{e}}, t)=\boldsymbol{x}_{d}^{(n)}-\boldsymbol{f}_{0}(\underline{\boldsymbol{x}})-\boldsymbol{G}_{0} \boldsymbol{u}(t)-\boldsymbol{l}(\underline{\boldsymbol{x}}(t), t)+\underline{\boldsymbol{K}}^{T} \underline{\boldsymbol{e}}=\boldsymbol{e}^{(n)}+\underline{\boldsymbol{K}}^{T} \underline{\boldsymbol{e}}$

If $(1 / 2) \boldsymbol{s}^{T}(\underline{\boldsymbol{e}}, t) \boldsymbol{s}(\underline{\boldsymbol{e}}, t)$ is chosen as a cost function, then its derivative is $\boldsymbol{s}^{T}(\underline{e}, t) \dot{\boldsymbol{s}}(\underline{e}, t)$. 


\subsection{The Robust Feedback Control System}

Since the FBECMAC cannot completely mimic an ideal controller, the approximation error induces a tracking error in the control system, so a robust controller is required, in order to make the control system stable. Thus, the control system is composed of a FBECMAC controller and a robust controller.

The proposed a FBECAMC control system for a nonlinear system is shown in Fig. 2 .

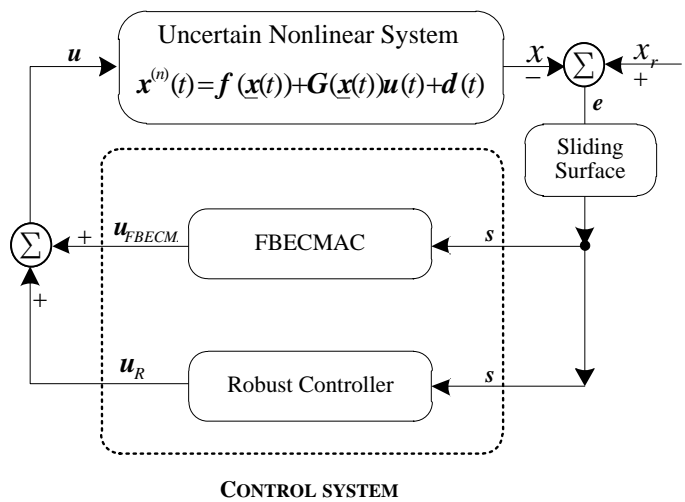

Figure 2

An intelligent control system for nonlinear systems

The control system is defined as:

$\boldsymbol{u}=\boldsymbol{u}_{F B E C M A C}+\boldsymbol{u}_{R}$

Substituting (25) into (24) and multiplying both sides by $\boldsymbol{s}^{T}(\underline{\boldsymbol{e}}, t)$ yields

$$
\begin{aligned}
\boldsymbol{s}^{T}(\underline{\boldsymbol{e}}, t) \dot{\boldsymbol{s}}(\underline{\boldsymbol{e}}, t)= & -\boldsymbol{s}^{T}(\underline{\boldsymbol{e}}, t) \boldsymbol{f}_{0}(\underline{\boldsymbol{x}}(t))-\boldsymbol{s}^{T}(\underline{\boldsymbol{e}}, t) \boldsymbol{G}_{0}\left[\boldsymbol{u}_{F B E C M A C}+\boldsymbol{u}_{R}(t)\right] \\
& +\boldsymbol{s}^{T}(\underline{\boldsymbol{e}}, t)\left(\boldsymbol{x}_{d}^{(n)}-\boldsymbol{l}(\underline{\boldsymbol{x}}(t), t)+\underline{\boldsymbol{K}}^{T} \underline{\boldsymbol{e}}\right)
\end{aligned}
$$

The fuzzy CMAC training algorithms in (20), (21) and (22) perform error back propagation, using the following chain-rule algorithm:

$$
\begin{aligned}
& \Delta w_{j q}=-\eta_{w} \frac{\partial \boldsymbol{s}^{T} \dot{\boldsymbol{s}}}{\partial w_{j q}}=-\eta_{w} \frac{\partial \boldsymbol{s}^{T} \dot{\boldsymbol{s}}}{\partial u_{F E C M A C}} \frac{\partial u_{F E C M A C}}{\partial w_{j q}}=\eta_{w} \cdot s_{i} g_{0 i}(x) \cdot\left[\prod_{i=1}^{n}\left(\phi_{i j}\left(I_{i}\right)\right)\right] \\
& \Delta \gamma_{i j}=-\eta_{m} \frac{\partial \boldsymbol{s}^{T} \dot{\boldsymbol{s}}}{\partial m_{i j}}=-\eta_{m} \frac{\partial \boldsymbol{s}^{T} \dot{\boldsymbol{s}}}{\partial u_{F E C M A C}} \frac{\partial u_{F E C M A C}}{\partial \phi_{i j}} \frac{\partial \phi_{i j}}{\partial \gamma_{i j}} \\
& =\eta_{m} \cdot s_{i} g_{0 i}(x) \cdot \sum_{q=1}^{p} w_{j q} \cdot\left[\prod_{i=1}^{n}\left(\phi_{i j}\left(I_{i}\right)\right)\right]\left(\frac{2\left(I_{i}-\gamma_{i j}\right)}{v_{i j}^{2}}\right)
\end{aligned}
$$




$$
\begin{aligned}
& \Delta v_{i j}=-\eta_{v} \frac{\partial \boldsymbol{s}^{T} \dot{\boldsymbol{s}}}{\partial v_{i j}}=-\eta_{v} \frac{\partial \boldsymbol{s}^{T} \dot{\boldsymbol{s}}}{\partial u_{F E C M A C}} \frac{\partial u_{F E C M A C}}{\partial \phi_{i j}} \frac{\partial \phi_{i j}}{\partial v_{i j}} \\
& =\eta_{v} \cdot s_{i} g_{0 i}(x) \cdot \sum_{q=1}^{p} w_{j q} \cdot\left[\prod_{i=1}^{n}\left(\phi_{i j}\left(I_{i}\right)\right)\right]\left(\frac{2\left(I_{i}-\gamma_{i j}\right)^{2}}{v_{i j}^{2}}\right)
\end{aligned}
$$

An approximation error between the FBECMAC and the ideal controller is navoidable, so an ideal controller is formulated as the summation of the FBECMAC and the approximation error:

$$
\boldsymbol{u}^{*}(t)=\boldsymbol{u}_{F B E C M A C}+\boldsymbol{\varepsilon}(t)
$$

where $\varepsilon(t)=\left[\varepsilon_{1}(t), \varepsilon_{2}(t), \ldots, \varepsilon_{m}(t)\right]^{T} \in \mathfrak{R}^{m}$ denotes the approximation error. It is assumed that $\|\varepsilon\| \leq E$, where $E$ is an unknown bound and $\|$.$\| is an induced norm.$ $\hat{E}$ is defined as an estimate of $E$, and $\tilde{E}=E-\hat{E}$.

From (6) and (24) and after some straightforward manipulations, it is seen that

$$
\boldsymbol{e}^{(n)}+\underline{\boldsymbol{K}}^{T} \underline{\boldsymbol{e}}=\boldsymbol{G}_{0}\left[\boldsymbol{u}^{*}-\boldsymbol{u}_{F B E C M A C}-\boldsymbol{u}_{R}(t)\right]=\boldsymbol{G}_{0}\left[\boldsymbol{\varepsilon}-\boldsymbol{u}_{R}\right]=\dot{\boldsymbol{s}}(\underline{\boldsymbol{e}}, t)
$$

Then the following theorem guarantees the stability of the feedback control system.

Theorem 1: For the $n$ th-order nonlinear systems represented by (3), the FBECMAC control system is designed as in (25), where $\boldsymbol{u}_{\text {FBECMAC }}$ is given in (16). The on-line parameter adaptation algorithms are given as (19)-(22) and the updating laws are given as (17) and (27)-(29), and the robust controller is designed as follows:

$\boldsymbol{u}_{R}=\hat{E} \tanh \left(\frac{s(t)}{\varsigma}\right)$

where $\tanh ($.$) is a hyperbolic tangent function, \hat{E}$ is the estimated value of the approximation error bound and $\varsigma$ is a positive parameter, such that:

$$
\dot{\hat{E}}=\eta_{\alpha}\left[s^{T}(\underline{e}, t) G_{0} \tanh \left(\frac{s(t)}{\varsigma}\right)-\xi\left(\hat{E}-E_{0}\right)\right]
$$

The feedback control system is then robustly stable.

Proof: The Lyapunov function is defined as:

$$
V(\boldsymbol{s}(\underline{\boldsymbol{e}}, t))=\frac{1}{2} \boldsymbol{s}^{T}(\underline{\boldsymbol{e}}, t) \boldsymbol{s}(\underline{\boldsymbol{e}}, t)+\frac{1}{2} \frac{\tilde{E}^{2}}{\eta_{\alpha}}
$$

The derivative of the Lyapunov function and (30) and (31) yield: 


$$
\begin{aligned}
\dot{V}(s(\underline{e}, t)) & =s^{T}(\underline{e}, t) \dot{\boldsymbol{s}}(\underline{e}, t)-\frac{\tilde{E} \dot{\hat{E}}}{\eta_{\alpha}} \\
& =\boldsymbol{s}^{T}(\underline{e}, t) \boldsymbol{G}_{0}\left[\varepsilon(t)-\boldsymbol{u}_{R}\right]-\frac{\tilde{E} \dot{\hat{E}}}{\eta_{\alpha}}
\end{aligned}
$$

The robust controller is designed as (32) and (33), so (35) can be rewritten as:

$$
\begin{aligned}
& \dot{V}(\boldsymbol{s}(\underline{e}, t)) \\
& =\boldsymbol{s}^{T}(\underline{\boldsymbol{e}}, t) \boldsymbol{G}_{0}\left[\boldsymbol{\varepsilon}(t)-\left(\hat{E} \tanh \left(\frac{\boldsymbol{s}(\underline{\boldsymbol{e}}, t)}{\varsigma}\right)\right)\right]-\frac{\tilde{E}}{\eta_{\alpha}} \eta_{\alpha}\left[\boldsymbol{s}^{T}(\underline{e}, t) \boldsymbol{G}_{0} \tanh \left(\frac{\boldsymbol{s}(\underline{\boldsymbol{e}}, t)}{\varsigma}\right)-\xi\left(\hat{E}-E_{0}\right)\right] \\
& =\boldsymbol{s}^{T}(\underline{\boldsymbol{e}}, t) \boldsymbol{G}_{0} \boldsymbol{\varepsilon}(t)-\boldsymbol{s}^{T}(\underline{\boldsymbol{e}}, t) \boldsymbol{G}_{0} \hat{E} \tanh \left(\frac{\boldsymbol{s}(\underline{\boldsymbol{e}}, t)}{\varsigma}\right)-\tilde{E} \boldsymbol{s}^{T}(\underline{\boldsymbol{e}}, t) \boldsymbol{G}_{0} \tanh \left(\frac{\boldsymbol{s}(\underline{\boldsymbol{e}}, t)}{\varsigma}\right)+\tilde{E} \xi\left(\hat{E}-E_{0}\right) \\
& =\boldsymbol{s}^{T}(\underline{\underline{e}}, t) \boldsymbol{G}_{0} \boldsymbol{\varepsilon}(t)-\boldsymbol{s}^{T}(\underline{\boldsymbol{e}}, t) \boldsymbol{G}_{0} \tanh \left(\frac{\boldsymbol{s}(\underline{\boldsymbol{e}}, t)}{\varsigma}\right)[\tilde{E}+\hat{E}]+\tilde{E} \xi\left(\hat{E}-E_{0}\right) \\
& \leq\left\|\boldsymbol { s } ^ { T } ( \underline { e } , t ) \left|\left\|\left|\boldsymbol{G}_{0}\right||| \mid \boldsymbol{\varepsilon}(t)\right\|-E \boldsymbol{s}^{T}(\underline{e}, t) \boldsymbol{G}_{0} \tanh \left(\frac{\boldsymbol{s}(\underline{\boldsymbol{e}}, t)}{\varsigma}\right)+\tilde{E} \xi\left(\hat{E}-E_{0}\right)\right.\right. \\
& \leq E\left[\left\|\boldsymbol{s}^{T}(\underline{\boldsymbol{e}}, t)\left|\left\||| \boldsymbol{G}_{0}\right\|-\boldsymbol{s}^{T}(\underline{\boldsymbol{e}}, t) \boldsymbol{G}_{0} \tanh \left(\frac{\boldsymbol{s}(\underline{\boldsymbol{e}}, t)}{\varsigma}\right)\right]+\tilde{E} \xi\left(\hat{E}-E_{0}\right)\right.\right.
\end{aligned}
$$

It is seen that the following inequality exists for any $\varsigma>0$ :

$$
0 \leq\left\|\boldsymbol{s}^{T}(\underline{\boldsymbol{e}}, t)\right\|\left\|\boldsymbol{G}_{0}\right\|-\boldsymbol{s}^{T}(\underline{\boldsymbol{e}}, t) \boldsymbol{G}_{0} \tanh \left(\frac{\boldsymbol{s}(\underline{\boldsymbol{e}}, t)}{\varsigma}\right) \leq \mho_{\varsigma}
$$

where $\vartheta$ is a constant that satisfies $\vartheta=\exp (-(\vartheta+1))$. Using inequality (37), (36) can be rewritten as:

$$
\begin{aligned}
\dot{V}(s(\boldsymbol{e}, t)) & \leq \xi \tilde{E}\left(\hat{E}-E_{0}\right)+E \vartheta \varsigma \\
& \leq-\frac{1}{2} \xi\left[(E-\hat{E})^{2}-\left(E-E_{0}\right)^{2}+\left(\hat{E}-E_{0}\right)^{2}\right]+E \vartheta \varsigma \\
& \leq-\frac{1}{2} \xi \tilde{E}^{2}+\frac{1}{2} \xi\left(E-E_{0}\right)^{2}+E \vartheta \varsigma
\end{aligned}
$$

Using the Lyapunov function (34), (38) can be rewritten as:

$\dot{V} \leq-\varpi V+\psi$

where $\varpi$ and $\psi$ are positive constants given by $\varpi=\xi \eta_{\alpha}$ 
$\psi=E \vartheta \varsigma+\frac{1}{2} \xi\left(E-E_{0}\right)^{2}$

Since $\frac{\psi}{\varpi}>0$ and the solution of the differential inequality satisfies

$$
0 \leq V(t) \leq \frac{\psi}{\varpi}+\left[V(0)-\frac{\psi}{\varpi}\right] e^{-\varpi t}
$$

where $V(0)$ is the initial value of $V$, then $s$ and $E$ are uniformly ultimately bounded, according to the extensions of the Lyapunov theory [15]. From (42), it is true that:

$$
\frac{1}{2} s^{2} \leq \frac{\psi}{\varpi}+\left[V(0)-\frac{\psi}{\varpi}\right] e^{-\varpi t} \leq \frac{\psi}{\varpi}+V(0) e^{-\varpi t}
$$

so

$$
s^{2} \leq 2\left[\frac{\psi}{\varpi}+V(0) e^{-\varpi t}\right]
$$

which implies that, given $\rho>\sqrt{2 \psi / \varpi}$, there exists a finite time, $T$, such that for all $t \geq T$, the tracking index satisfies:

$$
|s(\underline{e}, t)|<\rho
$$

where $\rho$ is the size of a small residual set that depends on the control system approximation error and the controller parameters and $\rho$ is a positive constant. $\rho$ is chosen to be small and the finite time is long, so that there is precise tracking of the error.

\section{Simulation Results}

Two uncertain nonlinear systems, a chaotic system and Mass-spring-damper mechanical system, are studied, in order to illustrate the effectiveness of proposed design.

\subsection{A Chaotic System}

For a general master-slave unified chaotic system, the master system is given as [16]:

$\dot{x}_{1}(t)=(25 \theta+10)\left(x_{2}(t)-x_{1}(t)\right)$ 
$\dot{x}_{2}(t)=(28-35 \theta) x_{1}(t)-x_{1}(t) x_{3}(t)+(29 \theta-1) x_{2}(t)$

$\dot{x}_{3}(t)=x_{1}(t) x_{2}(t)-\left(\frac{8+\theta}{3}\right) x_{3}(t)$

where $x_{i} i=1,2,3$ are the system state variables of the master system and $\theta=[0 \sim 1]$, where $\theta=[0 \sim 0.8)$, the system is known to be a generalized Lorenz system. When $\theta=0.8$, the system is called a Lu system, and when $\theta=(0 \sim 0.8]$, the system is called a Chen system. Figure 3 shows the state trajectories for $\theta=0$, with the initial condition: $x_{1}(0)=3, x_{2}(0)=5, x_{3}(0)=7$

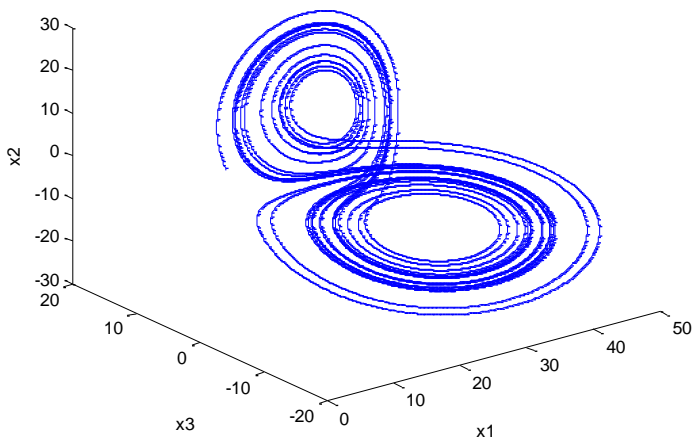

Figure 3

The unified chaotic system

It is assumed that $\pi_{1}=(25 \theta+10), \pi_{2}=(28-35 \theta), \pi_{3}=(29 \theta-1)$ and $\pi_{4}=\left(\frac{8+\theta}{3}\right)$, so (46) can be rewritten as:

$\dot{x}_{1}(t)=\pi_{1}\left(x_{2}(t)-x_{1}(t)\right)$

$\dot{x}_{2}(t)=\pi_{2} x_{1}(t)-x_{1}(t) x_{3}(t)+\pi_{3} x_{2}(t)$

$\dot{x}_{3}(t)=x_{1}(t) x_{2}(t)-\pi_{4} x_{3}(t)$

That is, the master system can be expressed as:

$\dot{\boldsymbol{x}}(t)=\boldsymbol{\pi}(\boldsymbol{x}(t))$

where $\boldsymbol{\pi}=\left[\pi_{1}, \pi_{2}, \pi_{3}\right]^{T} \quad \boldsymbol{x}(t)=\left[x_{1}(t), x_{2}(t), x_{3}(t)\right]^{T}$.

The slave system is given as:

$$
\begin{aligned}
& \dot{y}_{1}(t)=\pi_{1}\left(y_{2}(t)-y_{1}(t)\right)+\chi_{1}(t)+u_{1}(t) \\
& \dot{y}_{2}(t)=\pi_{2} y_{1}(t)-y_{1}(t) y_{3}(t)+\pi_{3} y_{2}(t)+\chi_{2}(t)+u_{2}(t)
\end{aligned}
$$


$\dot{y}_{3}(t)=y_{1}(t) y_{2}(t)-\varphi_{4} y_{3}(t)+\chi_{3}(t)+u_{3}(t)$

where $y_{i}, i=1,2,3$ are the system state variables of the slave system, $x_{i}, i=1,2,3$ are the external disturbances and $u_{i}, i=1,2,3$ are the control inputs.

This slave system can be also expressed as:

$\dot{\boldsymbol{y}}(t)=\boldsymbol{\pi}(y(t))+\boldsymbol{\zeta}(t)+\boldsymbol{u}(t)$

where $\quad \boldsymbol{y}(t)=\left[y_{1}(t), y_{2}(t), y_{3}(t)\right]^{T} \quad, \quad \boldsymbol{x}(t)=\left[x_{1}(t), x_{2}(t), x_{3}(t)\right]^{T} \quad$ and $\boldsymbol{u}(t)=\left[u_{1}(t), u_{2}(t), u_{3}(t)\right]^{T}$.

If the error states between the master system and the slave system are defined as:

$e_{1}(t)=y_{1}(t)-x_{1}(t)$

$e_{2}(t)=y_{2}(t)-x_{2}(t)$

$e_{3}(t)=y_{3}(t)-x_{3}(t)$

From (47) and (49) gives the error dynamics as:

$\dot{e}_{1}(t)=\pi_{1}\left(e_{2}(t)-e_{1}(t)\right)+x_{1}(t)+u_{1}(t)$

$\dot{e}_{2}(t)=\pi_{2} e_{1}(t)+\pi_{3} e_{2}(t)+x_{1}(t) x_{3}(t)-y_{1}(t) y_{3}(t)+x_{2}(t)+u_{2}(t)$

$\dot{e}_{3}(t)=y_{1}(t) y_{2}(t)-x_{1}(t) x_{2}(t)-\pi_{4} e_{3}(t)+x_{3}(t)+u_{3}(t)$

This can be also rewritten as:

$\dot{\boldsymbol{e}}(t)=\boldsymbol{A} \boldsymbol{e}(t)+\boldsymbol{f}(t)+\boldsymbol{x}(t)+\boldsymbol{u}(t)$

where $\boldsymbol{e}(t)=\left[e_{1}(t), e_{2}(t), e_{3}(t)\right]^{T}$ is the state error vector, $\boldsymbol{A}=\left[\begin{array}{ccc}-\pi_{1} & \pi_{1} & 0 \\ \pi_{2} & \pi_{3} & 0 \\ 0 & 0 & -\pi_{4}\end{array}\right]$, and $\boldsymbol{f}(t)=\left[\begin{array}{c}0 \\ x_{1}(t) x_{3}(t)-y_{1}(t) y_{3}(t) \\ -x_{1}(t) x_{2}(t)+y_{1}(t) y_{2}(t)\end{array}\right]$

In order to illustrate the effectiveness of the proposed FBECMAC control system, it is compared with the fuzzy neural network based controller in [16], The control parameters are selected as $k_{1}=\left[\begin{array}{ccc}0.1 & 0 & 0 \\ 0 & 0.1 & 0 \\ 0 & 0 & 0.2\end{array}\right], c_{q}=0.8, q=1,2,3, \eta_{z}=5 \times 10^{-4}$, $\eta_{w}=4, \eta_{m}=0.7, \eta_{v}=5 \times 10^{-3}, \varsigma=1$ and $\xi=2$ and the other parameters are 
random values. The fuzzy neural network $(\mathrm{FNN})$ control chaotic system is shown in Fig. 4. The tracking error for the FNN control system is shown in Fig. 5. The FBECMAC control chaotic system is shown in Fig. 6 and the tracking error for the FBECMAC control system is shown in Fig. 7. A comparison of the simulation results shows that the proposed FBECMAC control system achieves better control than a FNN control system.

\subsection{A Mass-Spring-Damper Mechanical System}

A mass-spring-damper mechanical system is shown in Fig. 8. The dynamic equations for this mechanical system are expressed as [16,17]:

$$
\begin{aligned}
& \tau_{1} \ddot{x}_{1}(t)=-f_{K 1}(x)-f_{B 1}(x)+f_{K 2}(x)+f_{B 2}(x)+u_{1}(t)+\Delta u_{12}(t)+\delta_{1}(t) \\
& \tau_{2} \ddot{x}_{2}(t)=-f_{K 2}(x)-f_{B 2}(x)+u_{2}+\Delta u_{21}+\delta_{2}(t)
\end{aligned}
$$

where $\tau_{1}$ and $\tau_{2}$ are the masses in the system and $\boldsymbol{x}(t)=\left[x_{1}(t), x_{2}(t), \dot{x}_{1}(t), \dot{x}_{2}(t)\right]^{T}$ are the positions and the velocities of the mechanical system. The spring forces are $f_{K 2}(\underline{x})=k_{20}\left(x_{2}-x_{1}\right)+\Delta k_{2}\left(x_{2}-x_{1}\right)^{3}$ and $f_{K 2}(\underline{x})=k_{20}\left(x_{2}-x_{1}\right)+\Delta k_{2}\left(x_{2}-x_{1}\right)^{3}$ and the frictional forces are $f_{B 1}(x)=b_{10} \dot{x}_{1}+\Delta b_{1} \dot{x}_{1}^{2}$ and $f_{B 2}(x)=b_{20}\left(\dot{x}_{2}-\dot{x}_{1}\right)+\Delta b_{2}\left(\dot{x}_{2}-\dot{x}_{1}\right)^{2}$. The parameters for the system are given as $\tau_{1}=1, \tau_{2}=0.8, \quad k_{10}=3 \quad, \quad k_{20}=4, \quad b_{10}=2, \quad b_{20}=2.2$, $\Delta k_{1}=0.5, \Delta k_{2}=0.5, \Delta b_{1}=0.5, \Delta b_{2}=0.5, \Delta u_{12}=0.2 u_{2}, \Delta u_{21}=0.25 u_{1}$,
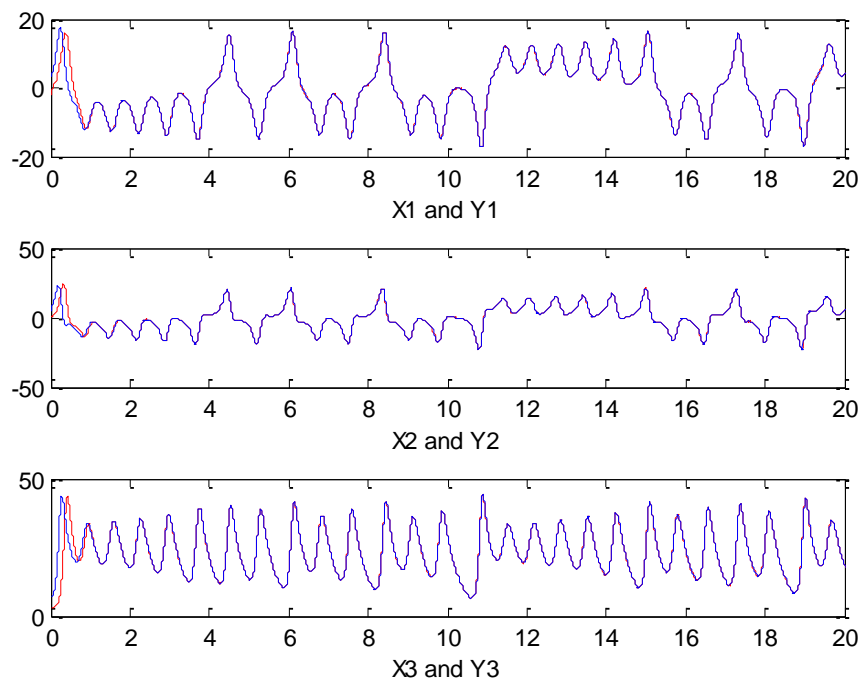

Figure 4

The FNN control for the chaotic system: (A) $x_{1}$ and $y_{1}$ (B) $x_{2}$ and $y_{2}$ (C) $x_{3}$ and $y_{3}$ 

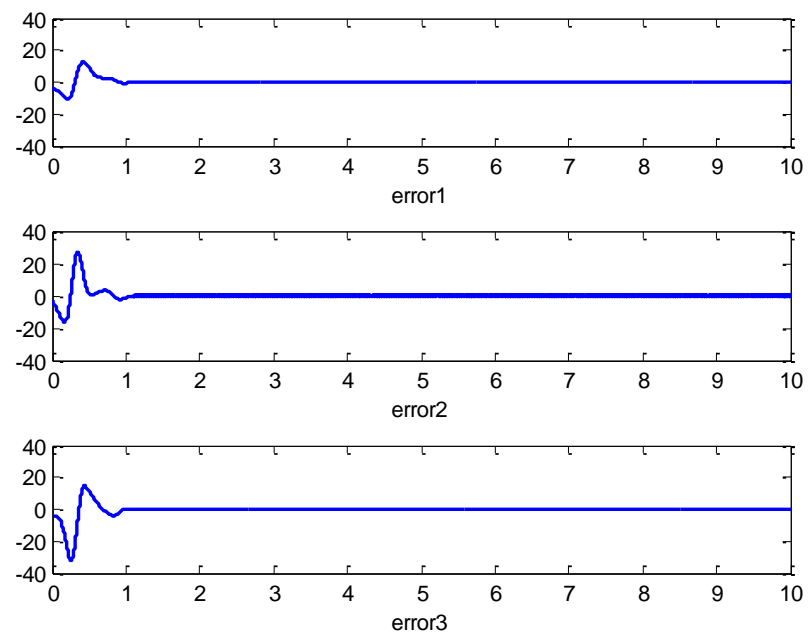

Figure 5

The tracking error for the FNN control system
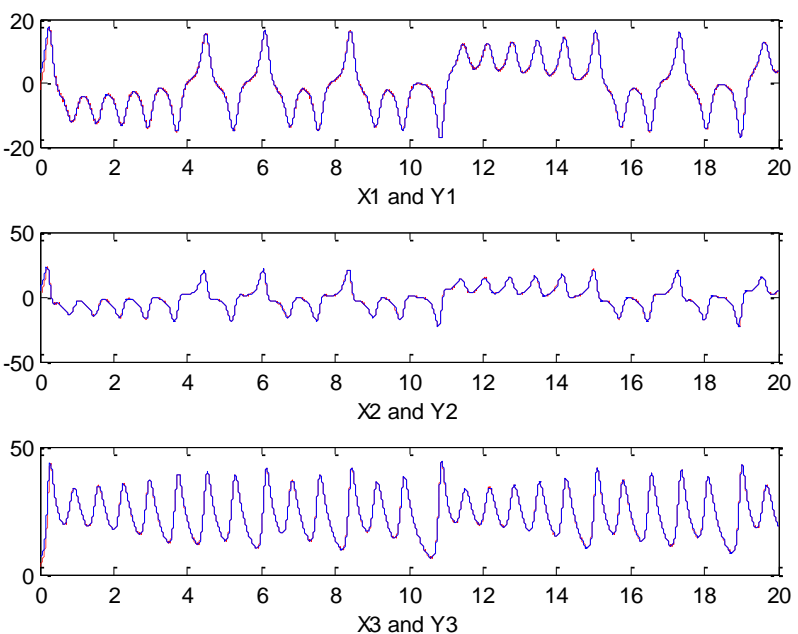

Figure 6

The FBECMAC control for the chaotic system: (A) $x_{1}$ and $y_{1}$ (B) $x_{2}$ and $y_{2}$ (C) $x_{3}$ and $y_{3}$ 

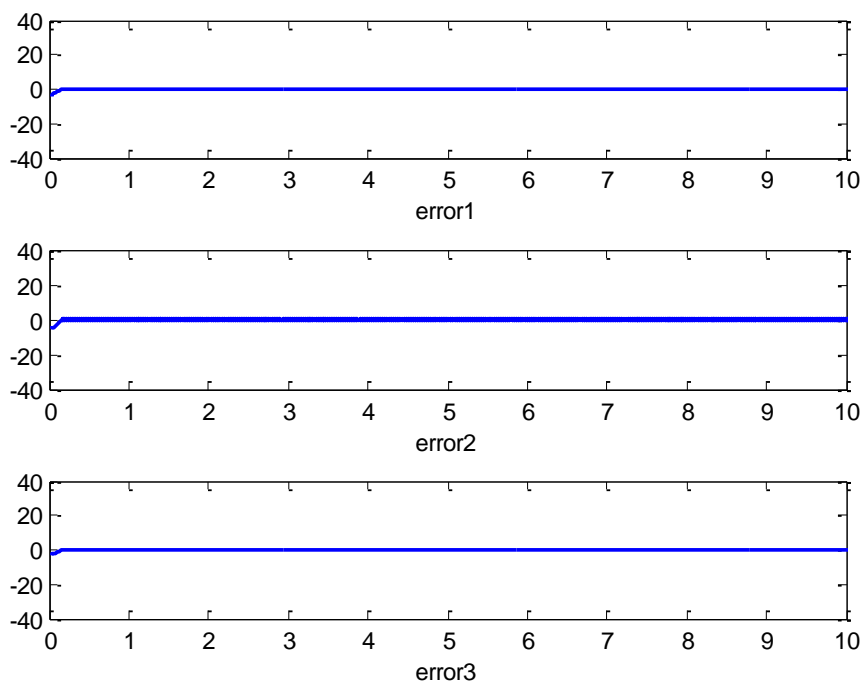

Figure 7

The tracking error for the FBECMAC control system

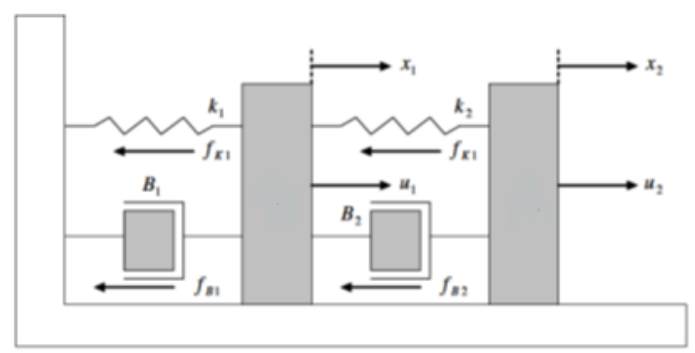

Figure 8

A mass-spring-damper mechanical system

$d_{1}(t)=2 \exp (-0.2 t)$ and $d_{2}(t)=-2 \exp (-0.1 t)$.

Consequently, the dynamic equation for the mass-spring-damper mechanical system can be rewritten as:

$\ddot{\boldsymbol{x}}(t)=\boldsymbol{f}(\underline{x})+\boldsymbol{G}(\underline{x}) \boldsymbol{u}(\mathrm{t})+\boldsymbol{d}(t)$

where

$$
f(\underline{x})=\left[\frac{-f_{K 1}(\underline{x})-f_{B 1}(\underline{x})+f_{K 2}(\underline{x})+f_{B 2}(\underline{x})}{\tau_{1}}, \frac{-f_{K 2}(\underline{x})-f_{B 2}(\underline{x})}{\tau_{2}}\right]
$$


$\boldsymbol{G}(\underline{x})=\left[\begin{array}{cc}\frac{1}{\tau_{1}} & \frac{0.2}{\tau_{1}} \\ \frac{0.25}{\tau_{2}} & \frac{1}{\tau_{2}}\end{array}\right]$ and $\boldsymbol{u}(t) \underline{\Delta}\left[u_{1}(t), u_{2}(t)\right]^{T}$ denotes the control input and $\boldsymbol{d}(t) \Delta\left[\frac{d_{1}(t)}{\tau_{1}}, \frac{d_{2}(t)}{\tau_{2}}\right]^{T}$ denotes the external disturbance. The desired trajectories come from the reference model outputs. The reference model is chosen as $\ddot{x}_{d i}(t)=-16 x_{d i}(t)-4 \dot{x}_{d i}(t)+12 r_{i}, i=1,2$. The initial conditions for the mechanical system and the reference model are given as $x_{1}(0)=1, \dot{x}_{1}(0)=0, x_{2}(0)=1$, $\dot{x}_{2}(0)=0, \quad x_{d 1}(0)=0, \quad x_{d 2}(0)=0, \dot{x}_{d 1}(0)=0$ and $\dot{x}_{d 2}(0)=0$. The control parameters are selected as $k_{1}=\left[\begin{array}{cc}0.01 & 0 \\ 0 & 0.01\end{array}\right], \quad c_{q}=0.8, q=1,2,3, \quad \eta_{z}=0.3$, $\eta_{w}=0.01, \eta_{m}=0.2, \eta_{v}=0.2, \varsigma=1.1$ and $\xi=1.5$ and the other parameters are random values. The reference inputs are $r_{1}=\frac{\pi}{3}\left(0.9 \sin \left(\frac{t}{2}\right)+0.1 \sin (2 t)\right)$ and $r_{2}=\pi(0.4 \sin (t)+0.1 \sin (3 t))$.

The FNN control for the mass-spring-damper mechanical system is shown in Fig. 9 and the tracking error is shown in Fig 10. The FBECMAC control for the massspring-damper mechanical system is shown in Fig. 11 and the tracking error is shown in Fig. 12. These simulations also demonstrate the better control of the FBECMAC control system.
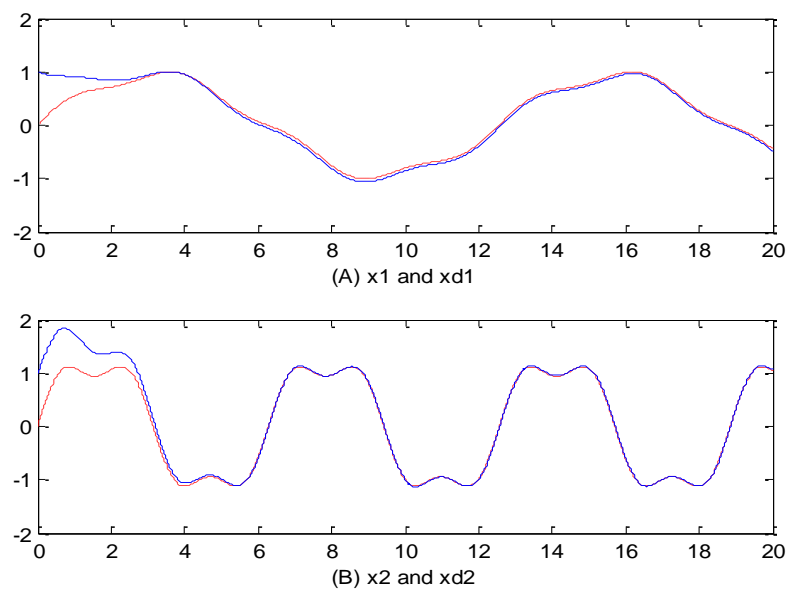

Figure 9

The FNN control for the mass-spring-damper mechanical system 

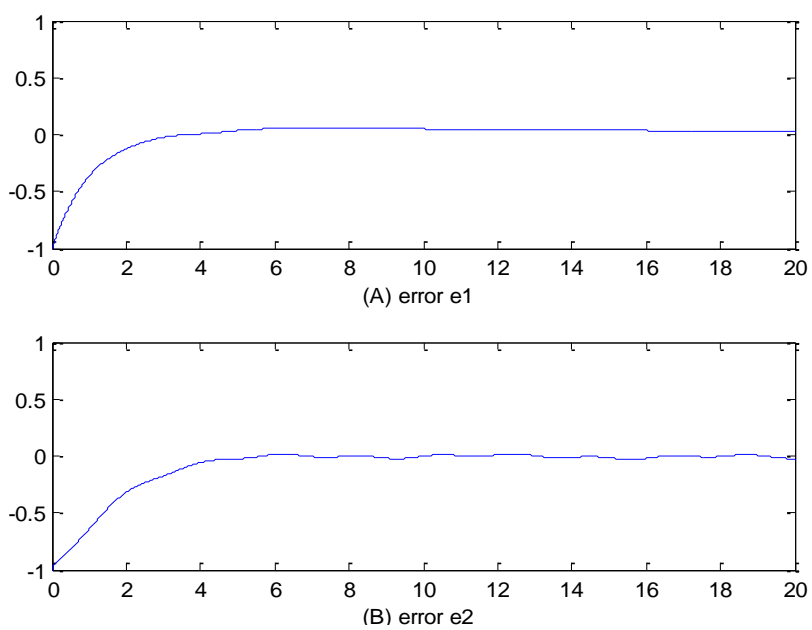

Figure 10

The tracking error for the FNN control system
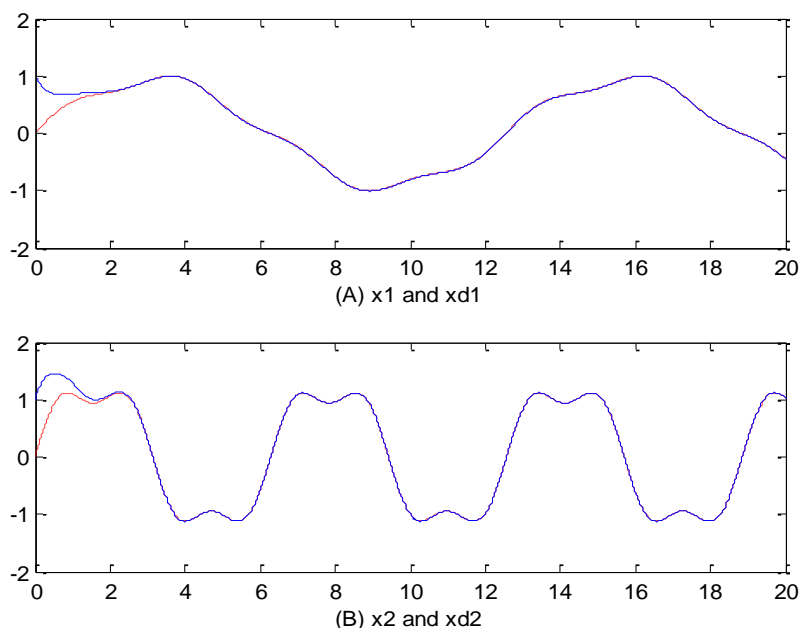

Figure 11

The FBECMAC control for the mass-spring-damper mechanical system 

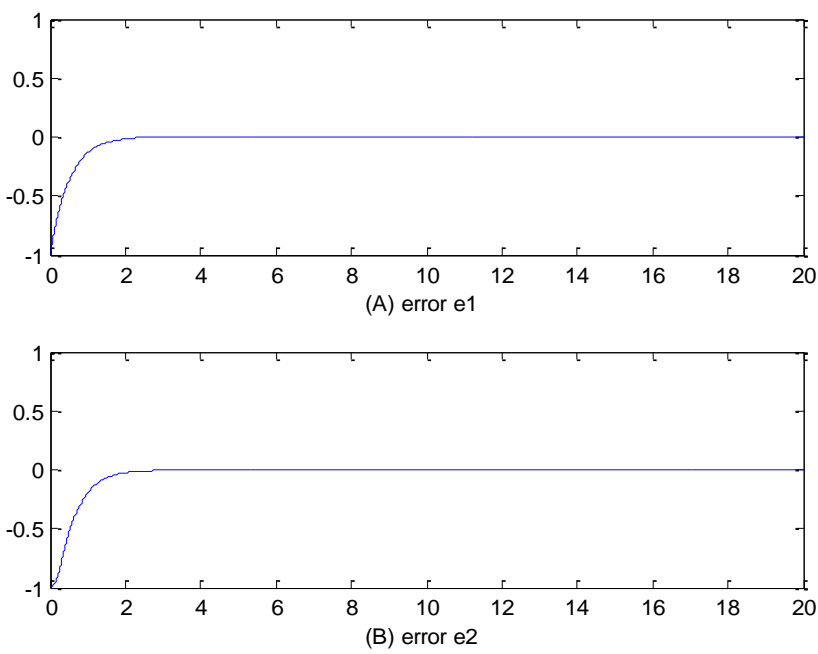

Figure 12

The tracking error for the FBECMAC control system

\section{Conclusion}

This study successfully proposes an efficient FBECMAC control system, which has the benefits of a fuzzy inference system and a brain emotional CMAC. The controller is then used to control nonlinear systems. The stability analysis is also presented in the feedback control system. The proposed FBECMAC reduces the tracking error, even if the systems are subjected to external disturbances. The results of the comparison also show that the tracking error converges faster in the FBECMAC than that in a fuzzy neural network control system.

\section{Acknowledgment}

This work was supported by the National Science Council of the Republic of China under Grant NSC-95-2221-E-155-014-MY3.

\section{Reference}

[1] J. E. LeDoux, "The Amygdala: Neurobiological Aspects of Emotion," Wiley-Liss, New York, pp. 339-351, 1992

[2] C. Balkenius and J. Moren, "Emotional Learning: A Computational Model of The Amygdala," Cybernetics and Systems, Vol. 32, No. 6, pp. 611-636, 2001

[3] J. Moren. "Emotion and Learning-A Computational Model of the Amygdala," PhD dissertation, Lund University, 2002

[4] M. Valikhani and C. Sourkounis, "A Brain Learning-based Intelligent Controller (BELBIC) for DFIG System," 2014 International Symposium on Power Electronics, Electrical Drives, Automation and Motion, pp. 713-718, 
2014

[5] M. A. Sharbafi, C. Lucas and R. Daneshavar, "Motion Control of OmniDirectional Three-Wheel Robots by Brain-Emotional-Learning-based Intelligent Controller," IEEE Transactions on Systems, Man, and Cybernetics, Part C: Applications and Reviews, Vol. 40, No. 6, pp. 630-638, 2010

[6] M. A. Rahman, R. M. Milasi, C. Lucas, B. N. Araabi and T. S. Radwan, "Implementation of Emotional Controller for Interior Permanent-Magnet Synchronous Motor Drive," IEEE Transactions on Industry Applications, Vol. 44, No. 5, pp. 1466-1476, 2008

[7] H. A. Zarchi, E. Daryabeigi, G. R. A. Markadeh and J. Soltani, "Emotional Controller (BELBIC) Based DTC for Encoderless Synchronous Reluctance Motor Drives," $20112^{\text {nd }}$ Power Electronics, Drive Systems and Technologies Conference, pp. 478-483, 2011

[8] J. S. Albus, “A New Approach to Manipulator Control: The Cerebellar Model Articulation Controller (CMAC)," Journal of Dynamic System, Measurement and Control, Vol. 97, No. 3, pp. 220-227, 1975

[9] C. M. Lin, C. F. Hsu and C. M. Chung, "RCMAC-based Adaptive Control Design for Brushless DC Motors," Neural Computing and Applications, Vol. 18, No. 7, pp. 781-790, 2009

[10] P. E. M. Almedia and M. G. Simoes, "Parametric CMAC Networks Fundamentals and Applications of a Fast Convergence Neural Structure," IEEE Transactions Industrial Applications, Vol. 39, No. 5, pp. 1551-1557, 2003

[11] C. M Lin and T. Y. Chen, "Self-Organizing CMAC Control for a Class of MIMO Uncertain Nonlinear System," IEEE Transactions on Neural Network, Vol. 20, No. 9, pp. 1377-1384, 2009

[12] C. M. Lin, Y. F. Peng and C. F. Hsu, "Robust Cerebellar Model Articulation Controller Design for Unknown Nonlinear Systems," IEEE Transactions on Circuits System, Vol. 51, No. 7, pp. 354-358, 2004

[13] L. Bessissa, L. Boukezzi and D. Mahi “ A Fuzzy Logic Approach to Model and Predict HV Cable Insulation Behaviour under Thermal Aging," Journal of Applied Science, Acta Polytechnica Hungarica, Vol. 11, No. 3, pp. 107-123, 2014

[14] C. B. Regaya, A. Zaafouri and A. Chaari, "A New Sliding Mode Speed Observer of Electric Motor Drive Based on Fuzzy- -Logic," Journal of Applied Science, Acta Polytechnica Hungarica, Vol. 11, No. 3, pp. 220-232, 2014

[15] J. J. E. Slotine and W. P. Li, "Applied Nonlinear Conrtol," Englewood Cloffs, NJ, USA:Prentice-Hall, 1991 
[16] C. M. Lin and C. F. Hsu, "Supervisory Recurrent Fuzzy Neural Network Control of Wing Rock for Slender Delta Wings," IEEE Trans. Fuzzy Systems, Vol. 12, No. 5, pp. 733-742, 2004

[17] Y. C. Chang, "Robust $H_{\infty}$ Control for a Class of Uncertain Nonlinear Time-Varying System and Its Application," IEE Proceedings, Control Theory and Applications, Vol. 151, No. 5, pp. 601-609, 2004 